\title{
Precision Tracking Algorithm Study of Surface-to-Air Missile on the Photodetector Matrix Forming Optics Glare Homing
}

\author{
Yury L. Koziratsky, Dmitri V. Prokhorov, \\ Igor Y. Kuryanov and Egor D. Shatsky* \\ Military Training and Research Center of the Air Force \\ «Air Force Academy ft. Professor N.E. Zhukovsky and Y.A. Gagarin» \\ 54a Starykh Bol'shevikov Str., Voronezh, 394064, Russia
}

Received 09.10.2015, received in revised form 26.11.2015, accepted 29.02.2016

In order to protect the aircraft from attacking surface-to-air missile with precision the algorithm of Activity-through the use of laser thermal imaging system with a receiver matrix type. As a discriminator considered algorithms assess the current position of the energy center of the image and the sum-difference signal processing, carrying out the measurement error angles between the direction of the surface-to-air missile and the optical axis of the matrix photodetector uneven coordinate sensitivity, noise immunity boosting algorithms. Developed a dynamic model of precision tracking, to evaluate the effectiveness of the formation at the entrance of the matrix image sensor in the form of an additive mixture of signal and noise.

Keywords: precision tracking, algorithm, optical electronic means.

Citation: Koziratsky Y.L., Prokhorov D.V., Kuryanov I.Y., Shatsky E.D. Precision tracking algorithm study of surface-toair missile on the photodetector matrix forming optics glare homing, J. Sib. Fed. Univ. Eng. technol., 2016, 9(3), 401-415. DOI: 10.17516/1999-494X-2016-9-3-401-415.

(c) Siberian Federal University. All rights reserved

* Corresponding author E-mail address: urleo@bk.ru 


\section{Исследование алгоритма}

высокоточного сопровождения

зенитных управляемых ракет

матричным фотоприемником по блику

формирующей оптики системы самонаведения

Ю.Л. Козирацкий, Д.В. Прохоров, И.Ю. Курьянов, Е.Д. Шатский

ВУНЦ ВВС «ВВА им. проф. Н.Е. Жуковского и Ю.А. Гагарина» Россия, 371600, Воронеж, ул. Старых Большевиков, 54 а

В интересах зашиты воздушного судна от атакующих зенитных управляемых ракет (ЗУР) предлагается алгоритм высокоточного сопровождения на основе использования лазерно-тепловизионной системы с приемным устройством матричного типа. В качестве дискриминатора рассмотрены алгоритмы оценки текущих координат энергетического центра изображения и суммарно-разностной обработки сигналов, осуществляющие измерения углов рассогласования между направлением на ЗУР и оптической осью матричного фотоприемника с неравномерной координатной чувствительностью, повышающей помехоустойчивость алгоритмов. Разработана динамическая модель прочесса высокоточного сопровождения, позволяющая оценить эффективность системы при формировании на входе матричного фотоприемника изображения в виде аддитивной смеси сигналов и помех.

Ключевые слова: высокоточное сопровождение, алгоритм, оптико-электронное средство.

\section{Введение}

Для успешной реализации перспективных способов защиты воздушного судна от поражения зенитными управляемыми ракетами (ЗУР) крайне важно решение задачи высокоточного сопровождения ЗУР [1-4]. Под высокоточным сопровождением будем понимать автоматическое сопровождение ЗУР с ошибками, среднее значение которых не превышает $5 \cdot 10^{-3}$ рад. Данному процессу, как правило, предшествуют этапы обнаружения, оценки текущих координат и нацеливания системы высокоточного сопровождения. Сущность процесса сопровождения заключается в отработке ошибки согласования оптической оси приемника и направления на ЗУР. Основным элементом системы высокоточного сопровождения, решающим эту задачу, как правило, является измеритель, включающий в себя приемник оптического излучения и дискриминатор. Для повышения точности сопровождение может осуществляться на основе комплексного использования пассивных и активных оптико-электронных средств (ОЭС). С целью повышения помехоустойчивости и точности сопровождения предлагается использовать матричный фотоприемник (МФП), осуществляющий измерения величины рассогласования энергетического центра изображения ЗУР и геометрического центра МФП. При этом предлагается использовать МФП с неравномерной координатной чувствительностью и формированием знакопеременных электрических сигналов. В случае нахождения изображения атакующей ЗУР в пределах фоточувствительной поверхности матричного фотоприемника можно определить его энергетический центр путем взвешенного суммирования величин сигналов по строкам и 
столбцам матричного приемника. В данном случае сущность процесса высокоточного сопровождения заключается в отработке ошибки (рассогласования центра матричного приемника и энергетического центра изображения ЗУР) таким образом, чтобы после повышения кратности увеличения формирующей оптики изображение атакующей ЗУР располагалось в пределах фоточувствительной поверхности, что достигается изменением фокусного расстояния.

Цель работы - синтез и анализ алгоритма высокоточного сопровождения ЗУР матричным фотоприемником лазерно-тепловизионной системы в условиях помеховых воздействий.

\section{Исследование приемного канала системы высокоточного сопровождения}

Основным каналом системы, обеспечивающим высокоточное сопровождение ЗУР, можно считать приемный канал на базе матричного приемника ИК-диапазона длин волн. Рассмотрим особенности построения и принцип функционирования приемного канала системы высокоточного сопровождения, структурная схема которого представлена на рис. 1. Приемная часть системы сопровождения двухканальная и обеспечивает сопровождение цели по двум составляющим угла рассогласования (углу курса и углу тангажа).

На вход объектива (оптической системы) приемной части лазерно-тепловизионной системы попадает оптическое излучение, представляющее собой трехмерный сигнал в виде аддитивной смеси полезного отраженного от головки самонаведения $(\Gamma \mathrm{CH})$ зондирующего сигнала лазерного излучения, отраженного от корпуса ЗУР зондирующего лазерного излучения, ИКизлучения факела маршевого двигателя ЗУР, фонового излучения и излучения, обусловленного действием преднамеренных помех:

$$
E_{\Sigma \lambda}(x, y, t)=E_{\sigma \lambda}(x, y, t)+E_{\text {omp } \lambda}(x, y, t)+E_{\phi \partial \lambda}(x, y, t)+E_{\phi \lambda}(x, y, t)+E_{n \lambda}(x, y, t),
$$

где $E_{\lambda}(x, y, t)$ - спектральные плотности энергетической освещенности одного элемента фоточувствительной поверхности матричного фотоприемника с координатами $x$ и $y$, обусловленные воздействием вышеуказанных излучений [5].

При этом положение энергетического центра полезного сигнала изображения на плоскости МФП (относительно центра его координатной системы) характеризует угол рассогласования между оптической осью объектива приемника и направлением на ГСН ЗУР. В результате прохождения сигнала через формирующую оптику объектива (оптическую систему) на плоскости МФП формируется изображение. Далее в соответствии с алгоритмом оценки смещения энергетического центра изображения ЗУР относительно геометрического центра МФП дискриминатор формирует напряжения рассогласования $U_{x}$ и $U_{y}$, пропорциональные отклонению энергетического пятна по углу курса и тангажа соответственно. Выработанные координатные импульсы (сигналы $U_{x}$ и $U_{y}$ ), связанные с системой координат МФП, поступают на формирователи сигналов поворота, осуществляющие формирование импульсов необходимой длительности, обеспечивающей вращение роторов серводвигателей на необходимое число оборотов. Сформированные сигналы подаются на усилители мощности (УМ), на которые поступают и сигналы со счетчиков угла поворота. Счетчики угла поворота осуществляют подсчет числа оборотов роторов серводвигателей и обеспечивают коррекцию поступающих на вход усилителей мощности сигналов для обеспечения точного срабатывания серводвигателей в направлении

$$
-403-
$$




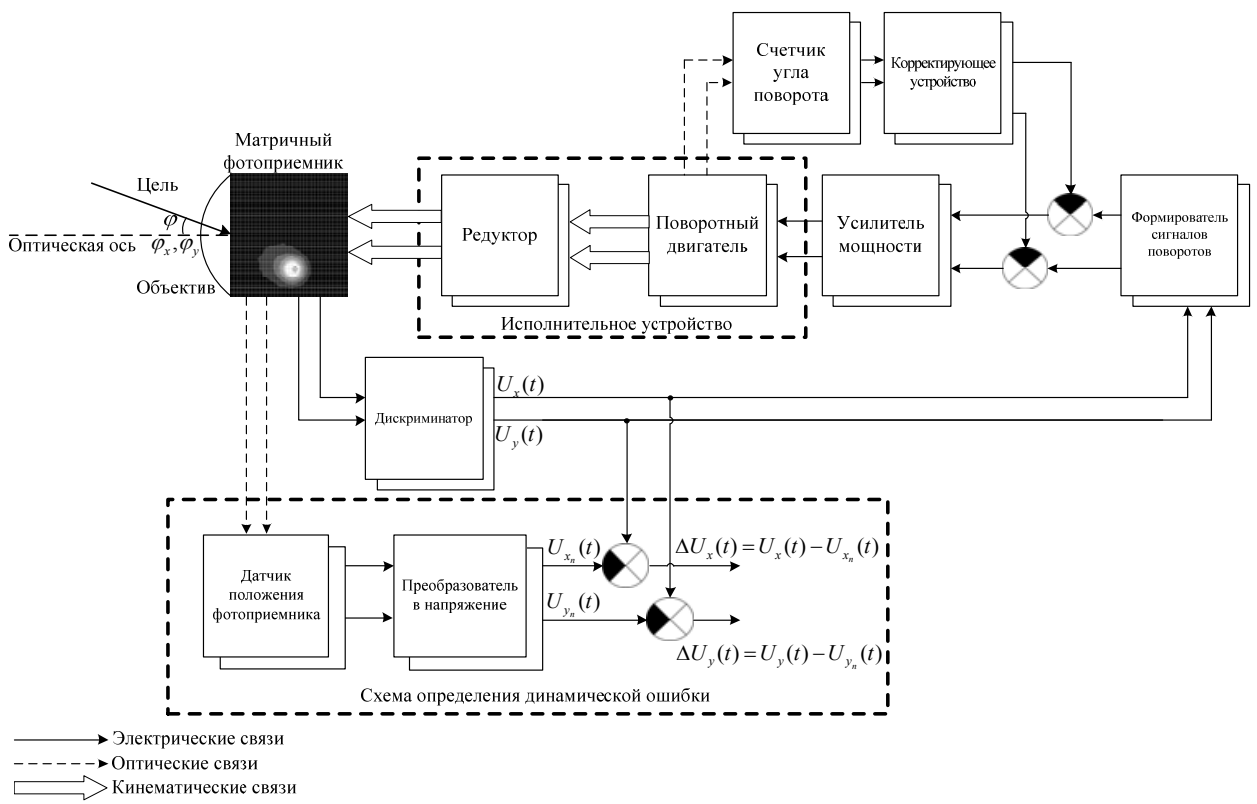

Рис. 1. Структурная схема приемной части системы высокоточного сопровождения ЗУР

уменьшения угла рассогласования. Усилители мощности вводятся для обеспечения нормальной работы электрических двигателей, которые через редукторы с некоторым передаточным числом $i$ поворачивают объектив (следящее зеркало) [3] в направлении уменьшения угла рассогласования. В результате происходит коррекция ориентирования оси оптической системы в сторону цели, уменьшая угол рассогласования между осью объектива МФП и направлением на цель.

Как показал анализ работ [4-8], в основу перспективных средств сопровождения закладывается суммарно-разностный принцип обработки изображений при сопровождении движущейся ракеты и алгоритм оценки текущих координат энергетического центра изображения цели в плоскости матричного фотоприемника.

В данных алгоритмах сопровождение осуществляется по двум каналам: каналу сопровождения по углу курса $(x)$ и каналу сопровождения по тангажу $(y)$. Каналы идентичны по своему составу. Особенностью высокоточного сопровождения в данном случае является то, что используется флюгерный принцип, сущность которого состоит в том, что формирующая оптика вместе с матричным приемником оптико-электронного измерителя сопровождают ракету с ошибками, не превышающими заданного уровня.

\section{Исследование эффективности алгоритма оценки}

\section{смещения энергетического центра пуассоновского изображения цели}

\section{в плоскости матричного фотоприемника}

Рассмотрим задачу разработки алгоритма функционирования приемника лазерного локатора при обнаружении и оценке смещения изображения цели в плоскости матричного фотоприемника $[7,8]$. 
Пусть входное воздействие на фоточувствительной поверхности (рисунок 2) представляется в виде

$$
\xi(x, y, t)=S(x, y, t)+n(x, y, t)
$$

где $\xi(x, y, t)$ - принимаемое (после компенсации) изображение; $S(x, y, t)$ - полезное изображение; $n(x, y, t)$ - фоновое изображение (после компенсации) и внутренние шумы, пересчитанные на вход приемника.

Интервал наблюдения $\left[t_{0}, t_{0}+T\right]$ ( $T$ - длительность интервала наблюдения фотоотсчетов) разобьем на равноотстоящие точки $t_{1}, t_{2}, \ldots, t_{k}, \ldots, t_{K}$, причем $t_{k}-t_{k-1}=\Delta_{2}=$ const $\left(\mathrm{T} / \Delta_{2}=K\right.$, где $\Delta_{2}$ - интервал дискретизации по времени).

Размеры элементарных приемников удовлетворяют условиям

$$
\Delta S=\left(x_{i}-x_{i-1}\right)\left(y_{j}-y_{j-1}\right)=\Delta x \Delta y=\Delta_{1} \Delta_{1}=\Delta_{1}^{2},
$$

где $\Delta S$ - чувствительная площадь поверхности элементарного приемника.

Число фотоэлектронов (образования переходов) в элементарном приемнике, имеющем координаты $\left(x_{i}, y_{i}\right)$, за один отсчет

$$
\xi_{i j}(k)=\frac{\eta}{h v} \int_{t_{k}-\Delta_{2} x_{i}-\Delta_{1}}^{t_{t}} \int_{y_{j}-\Delta_{1}}^{x_{i}} \int_{j}^{y_{j}} \xi(x, y, t) d y d x d t \simeq \gamma_{\xi_{j}} \Delta_{2}
$$

где $\gamma_{\xi_{i j}}=\frac{\eta}{h v} \int_{x_{i}-\Delta_{1}}^{x_{i}} \int_{y_{j}-\Delta_{1}}^{y_{j}} \xi(x, y, t) d y d x$.

Поступая аналогично к полезному и фоновому изображениям, получим:

$$
S_{i j}(k)=\frac{\eta}{h v} \int_{t_{k}-\Delta_{2}}^{t_{k}} \int_{x_{i}-\Delta_{1}}^{x_{i}} \int_{y_{j}-\Delta_{1}}^{y_{j}} S(x, y, t) d y d x d t \simeq \gamma_{s_{i j}} \Delta_{2}
$$

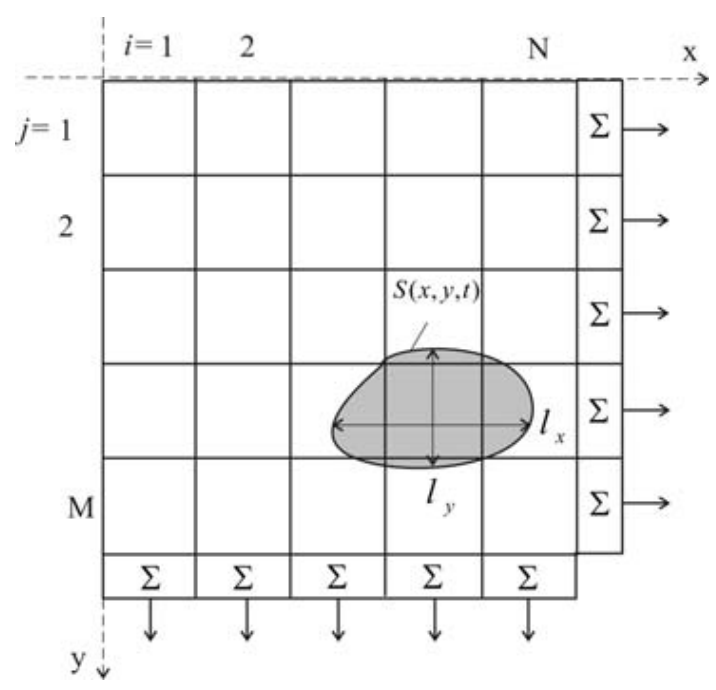

Рис. 2. Изображение на входе матричного фотоприемника

$$
-405-
$$




$$
n_{i j}(k)=\frac{\eta}{h v} \int_{t_{k}-\Delta_{2} x_{i}-\Delta_{1}}^{t_{k}} \int_{y_{j}-\Delta_{1}}^{x_{i}} n_{\phi}(x, y, t) d y d x d t+\int_{t_{k}-\Delta_{2}}^{y_{k}} n_{6}(t) d t \simeq \gamma_{n_{i j}} \Delta_{2}
$$

Тогда выражение (2) можно представить в виде

$$
\xi_{i j}(k)=S_{i j}(k)+n_{i j}(k),
$$

где $i=1,2, \ldots, N ; j=1,2, \ldots, M ; k=1,2, \ldots, K(i-$ индекс, относящийся к дискретизации по координате $x ; j$ - по координате $y ; k$ - по времени).

Определим оптимальные алгоритмы приемника при оценке смещения изображения по оси $x-\lambda_{x}$, по оси $y-\lambda_{y}$. Положим, что оценка будет проводиться на основе метода максимума апостериорной вероятности. Использование метода максимума апостериорной вероятности предполагает тот факт, что должны быть известны априорные плотности вероятностей оцениваемых параметров и пространственно-временная форма изображения.

Пространственно-временная форма импульса (изображения) в предположении гауссовского распределения [2] может быть записана таким образом:

$$
S(x, y, t)=\frac{2 \gamma(t)}{\pi l_{x} l_{y}} e^{-\frac{x^{2}}{l_{x}^{2} / 2}} e^{-\frac{y^{2}}{l_{y}^{2} / 2}} ; \gamma(t)=\gamma_{0} \frac{T_{u}}{\sqrt{2 \pi} \frac{W_{u}}{2}} e^{-\frac{t^{2}}{W_{u}^{2} / 2}},
$$

где $l_{x}$ и $l_{y}$ - размеры изображения по осям ох и оу соответственно; $W_{u}$ - ширина временной формы импульса; $T_{u}$ - период повторения импульса; $\gamma_{0}$ - среднее значение $\gamma(t)$.

Для случая гауссовских априорных плотностей вероятности оцениваемых параметров в соответствии с [2] применительно к матричному приемнику можно записать следующие выражения для оценки соответствующих параметров:

$$
\hat{\lambda}_{x}=\frac{\Delta_{1} \sum_{i=1}^{N} i \xi\left(i \Delta_{1}, t\right)+\frac{m_{x} l_{x}^{2}}{4 \Delta_{1} \sigma_{x}^{2}}}{\sum_{i=1}^{N} \xi\left(i \Delta_{1}, t\right)+\frac{l_{x}^{2}}{4 \Delta_{1} \sigma_{x}^{2}}} ; \hat{\lambda}_{y}=\frac{\Delta_{1} \sum_{j=1}^{M} j \xi\left(j \Delta_{1}, t\right)+\frac{m_{y} l_{y}^{2}}{4 \Delta_{1} \sigma_{y}^{2}}}{\sum_{j=1}^{M} \xi\left(j \Delta_{1}, t\right)+\frac{l_{y}^{2}}{4 \Delta_{1} \sigma_{y}^{2}}},
$$

где $m_{x}, m_{y}, m_{\tau}$ и $\sigma_{x}^{2}, \sigma_{y}^{2}, \sigma_{\tau}^{2}$ - математические ожидания и дисперсии гауссовских априорных плотностей вероятности оцениваемых параметров.

Полученные выражения (9) представляют собой искомые оптимальные алгоритмы приемника при оценке смещения изображения по оси $x-\lambda_{x}$, по оси $y-\lambda_{y}$.

На рис. 3 представлена схема устройства оценки смещения энергетического центра изображения, синтезированная на основе разработанных алгоритмов (9). На схеме введены обозначения:

$$
B_{x 1}=\frac{m_{x} l_{x}^{2}}{4 \Delta_{1} \sigma_{x}^{2}} ; B_{y 1}=\frac{m_{y} l_{y}^{2}}{4 \Delta_{1} \sigma_{y}^{2}} ; B_{x 2}=\frac{l_{x}^{2}}{4 \Delta_{1} \sigma_{x}^{2}} ; B_{y 2}=\frac{l_{y}^{2}}{4 \Delta_{1} \sigma_{y}^{2}}
$$

Полученные оценки координат энергетического центра изображения ЗУР (с учетом переноса начала системы координат) поступают в блок формирования управляющего напряжения, формирующий электрические сигналы рассогласования $U_{x}$ и $U_{y}$. 


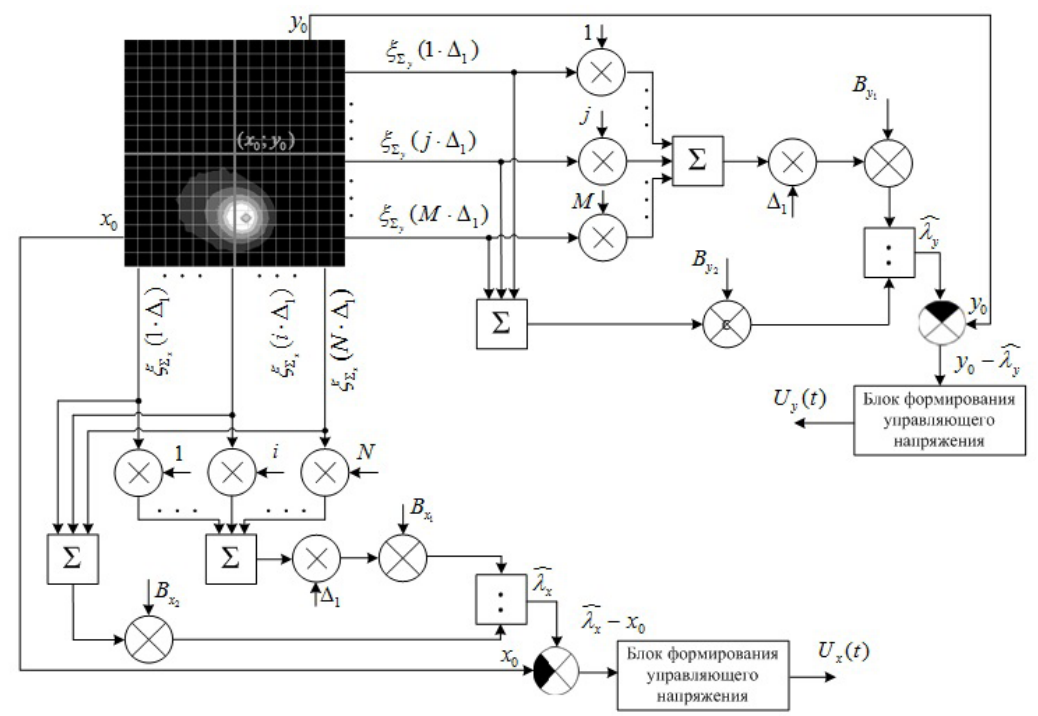

Рис. 3. Схема дискриминатора на основе алгоритма оценки смещения энергетического центра изображения

Если за начало выбран центр матричного приемника $\left(x_{0}=N / 2 ; y_{0}=M / 2\right)$, то величина отклонения энергетического центра изображения ЗУР от выбранного отсчета будет определяться парой уравнений: $\Delta x=\widehat{x}_{3}=\hat{\lambda}_{x}-\frac{N}{2} ; \quad \Delta \mathrm{y}=\hat{y}_{3}=\frac{M}{2}-\hat{\lambda}_{y}$.

Сформированное таким образом рассогласование, вызванное отклонением энергетического центра от выбранных отсчетных значений, уже с учетом знака полученной величины может служить для точного сопровождения ЗУР.

Следовательно, сигнал на выходе измерителя по соответствующему каналу будет равен величине $\hat{\lambda}_{y}$, а величина рассогласования, соответствующая координате $\hat{\lambda}_{y}, \Delta y=M / 2-\hat{\lambda}_{y}$.

Поэтому пеленгационная характеристика в традиционном понимании может быть представлена в виде следующей функциональной зависимости:

$$
\Delta y=f\left(M / 2-\hat{\lambda}_{y}\right) .
$$

При определении пеленгационной характеристики должен учитываться знак разницы $\frac{M}{2}-\hat{\lambda}_{y}$, который необходим для отработки возникшего рассогласования (ошибки). Если знак положительный, то смещение изображения должно производиться в сторону увеличения значений $j$ (вниз), в случае отрицательного знака, наоборот, в сторону уменьшения значений $j$ (вверх). Если заложен “флюгерный” принцип сопровождения ЗУР, то по отношению к приемнику положительный знак соответствует повороту вверх, а отрицательный - вниз. На рис. 4 представлены зависимости математического ожидания и среднеквадратического отклонения ошибок оценок координат энергетического центра изображения по осям $x$ и $y$ от отношения сигнал-шум q. Анализ полученных результатов показывает, что при уменьшении отношения сигнал-шум оценка положения цели смещается к центру матрицы, а ошибка определения положения изображения цели определяется удалением истинного положения цели от центра матричного фотоприемника. 




a)

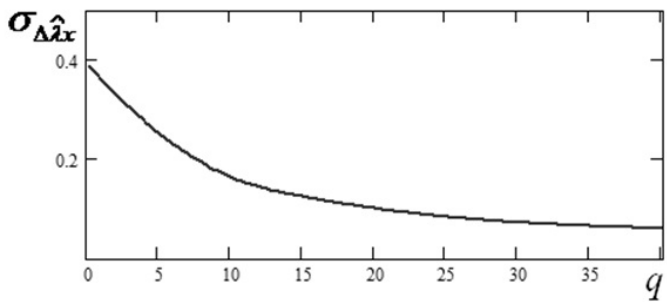

б)

Рис. 4. Зависимости от отношения сигнал - шум: $a$ - математического ожидания ошибок оценок координат энергетического центра изображения по осям $x$ и $y$; $\sigma$ - среднеквадратического отклонения ошибок оценок координат энергетического центра изображения по оси $x$

\section{Исследование эффективности алгоритма оценки} рассогласования энергетического центра изображения цели и геометрического центра матричного фотоприемника на основе суммарно-разностной обработки сигналов с квадрантов матричного фотоприемника

Рассмотрим алгоритм оценки рассогласования энергетического центра изображения цели и геометрического центра матричного фотоприемника на основе суммарно-разностной обработки (СРО) сигналов $[4,6]$. Четыре идентичных чувствительных площадки матричного фотоприемного устройства размещены в фокальной плоскости объектива так, что линии раздела между квадрантами образуют декартову систему координат (рис. 5). Изображение цели попадает на чувствительные площадки МФП. Далее импульсы, принятые МФП, преобразуются в схеме суммарно-разностной обработки. Принцип действия такой схемы заключается в том, что поток излучения в зависимости от смещения объекта относительно оптической оси попадает на четыре квадранта МФП. Каждый из формируемых квадрантами сигналов пропорционален площади, занимаемой изображением на соответствующей площадке. Сигналы с каждого из квадрантов суммируются (рис. 5) и далее сравниваются между собой. Разностный сигнал в этом случае пропорционален величине смещения изображения относительно нулевого положения.

В двух делителях результаты разностных устройств делятся на результат суммирования напряжения с квадрантов. В итоге образуются сигналы

$$
U_{x}=\frac{\left(U_{1}+U_{4}\right)-\left(U_{2}+U_{3}\right)}{U_{1}+U_{2}+U_{3}+U_{4}} ; U_{y}=\frac{\left(U_{1}+U_{2}\right)-\left(U_{3}+U_{4}\right)}{U_{1}+U_{2}+U_{3}+U_{4}},
$$

где $U_{1}=\frac{\eta е}{h v} P_{1}-$ напряжение (на единичном сопротивлении) на выходе первого квадранта; $P_{1}$ - мощность излучения, падающего на первый квадрант МФП.

\section{Способ повышения помехоустойчивости}

и чувствительности системы сопровождения

Предварительные оценки эффективности рассмотренных алгоритмов показали их недостаточную устойчивость к помеховым воздействиям, приводящим к большим ошибкам в определении величины рассогласования энергетического центра принимаемого изображения и 


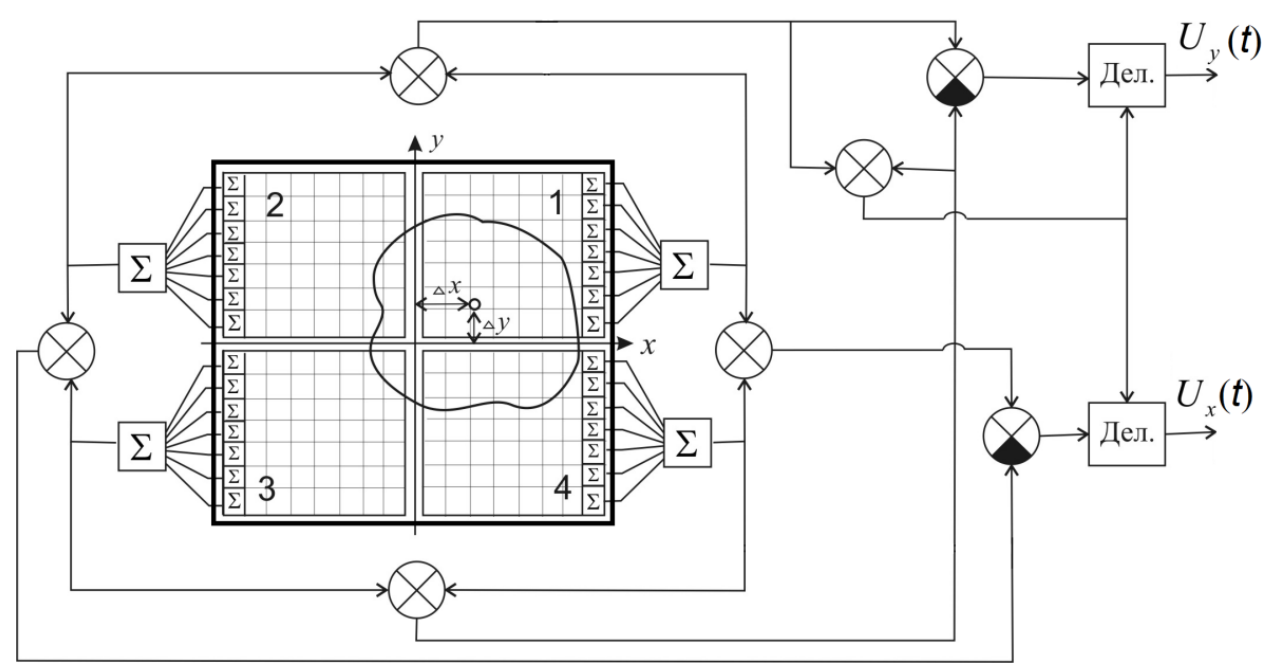

Рис. 5. Схема дискриминатора на основе суммарно-разностной обработки сигналов с квадрантов матричного фотоприемника

геометрического центра МФП. При этом ошибки в определении положения изображения цели тем больше, чем больше удаление истинного положения цели от центра МФП, что влияет на точность сопровождения и обуславливает возможность его срыва, учитывая динамику полета ракеты.

Поэтому возникает необходимость в разработке способов повышения помехоустойчивости и чувствительности системы сопровождения на основе рассмотренных алгоритмов.

Сущность предлагаемого способа заключается в применении переменных коэффициентов усиления для элементов матричного фотоприемника, увеличивающихся пропорционально удалению их от центра матрицы [9].

С этой целью могут использоваться матричные приемники с неравномерной координатной чувствительностью или методы пространственной фильтрации.

Рассмотрим сущность способа на примере использования методов пространственной фильтрации [10]. Принимаемое МФП изображение $\xi(x, y)$ до обработки в рассмотренных выше алгоритмах подлежит обработке с использованием специальной маски посредством операции типа «умножение одного изображения на другое» [10], осуществляя поэлементное перемножение изображения $\xi(x, y)$ и маски $w(x, y)$ размерностью $N \times M$ (равной размерности матричного фотоприемника):

$$
g(x, y)=\xi(x, y) \cdot w(x, y) .
$$

В качестве $w(x, y)$ предлагается взять следующую маску:

$$
w(x, y)=a \cdot\left(1-e^{-\frac{\left(x-\frac{N}{2}\right)^{2}}{2 \sigma_{1}^{2}}} \cdot e^{-\frac{\left(y-\frac{M}{2}\right)^{2}}{2 \sigma_{2}^{2}}}\right)+b,
$$

$$
-409-
$$


где $a$ - коэффициент, определяющий максимальные значения множителя (для элементов по периметру матричного фотоприемника); $b$ - коэффициент, определяющий минимальное значение множителя (для центра маски); $\sigma_{1}$ и $\sigma_{2}$ - среднеквадратические отклонения (для квадратной матрицы $\left.\sigma_{1}=\sigma_{2}=\sigma\right) ; N$ и $M$ - число элементов матричного фотоприемника по оси $x$ и $y$ соответственно.

В результате поэлементного перемножения изображения $\xi(x, y)$ и маски $w(x, y)$ имеем изображение $g(x, y)$, подлежащее последующей обработке в соответствии с вышерассмотренными алгоритмами. Коэффициенты элементов маски, увеличивающиеся от значения $b$ в центре к максимальным значениям по периметру в соответствии с двумерным гауссовским законом, обеспечивают компенсацию ошибок в определении энергетического центра изображения $\xi(x, y)$, и в особенности целей, удаленных от центра МФП, а также повышают крутизну пеленгационной характеристики, а следовательно, и чувствительность системы сопровождения.

\section{Разработка динамической модели процесса высокоточного сопровождения ЗУР лазерно-тепловизионной системой}

Для исследования эффективности алгоритма высокоточного сопровождения ЗУР матричным фотоприемником лазерно-тепловизионной системы необходима разработка динамической модели процесса высокоточного сопровождения.

Регулируемым параметром в системе сопровождения ЗУР является угол отклонения оси формирующей оптики МФП от направления на ГСН атакующей ЗУР, а управляющим воздействием (входным сигналом) - напряжения рассогласования, формируемые дискриминатором и обусловленные рассогласованием оптической оси МФП и направлением на ГСН ЗУР в двух плоскостях. Объект управления - объектив (оптическая система) системы сопровождения, состояние которой должно изменяться так, чтобы ее оптическая ось с необходимой точностью была направлена на сопровождаемую цель (ГСН ЗУР).

По своей структуре система сопровождения - система автоматического регулирования с отрицательной обратной связью $[3,11]$. Проектирование таких систем должно выполняться на базе общей теории управления.

Основные задачи исследования замкнутого контура системы сопровождения ЗУР - определение устойчивости контура и анализ ошибок управления и наведения. На основе исследования контура должны быть установлены оптимальные передаточные функции отдельных звеньев системы управления. Контур модели системы сопровождения ЗУР с передаточными функциями звеньев, полученный в соответствии со структурной схемой (см. рис. 1), представлен на рис. 6.

Входным воздействием $x(t)$ в нашем случае будет напряжение рассогласования, формируемое дискриминатором и обусловленное рассогласованием оптической оси МФП и направлением на ГСН ЗУР в соответствующей плоскости $U_{x}(t)\left(U_{y}(t)\right)$, а выходным $y(t)$ - напряжение рассогласования на выходе системы, обуславливающее корректировку текущего положения оптической системы в пространстве $U_{x n}(t)\left(U_{y n}(t)\right)$.

Считая элементы схемы (см. рис. 1) линейными звеньями, на схеме (рис. 6) введены следующие обозначения передаточных функций:

$$
-410-
$$




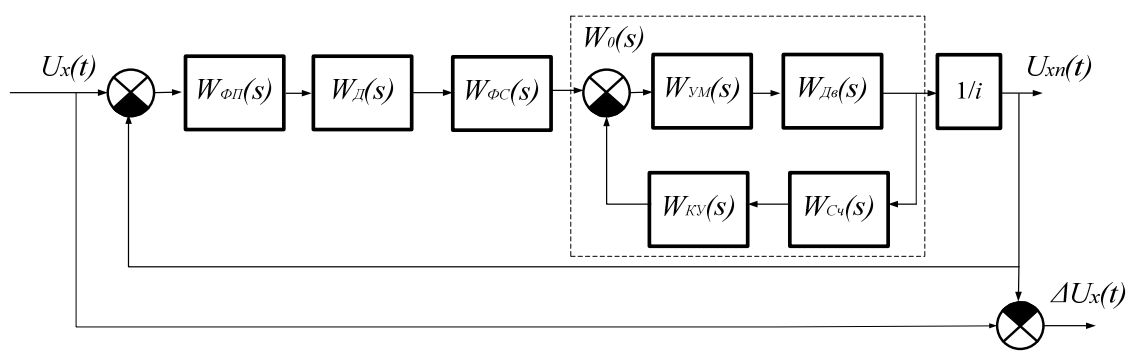

Рис. 6. Структурная схема имитационной модели процесса высокоточного сопровождения ЗУР лазернотепловизионной системой

$$
\begin{aligned}
& W_{\text {ФI }}(s)=\frac{k_{\phi \Pi}}{1+s T_{\phi \Pi}}-\text { передаточная функция МФП }\left(k_{\phi \Pi}=1\right) \text {; } \\
& W_{\text {д }}(s)=\frac{k_{\text {Д }}}{1+s T_{\text {д }}}-\text { передаточная функция дискриминатора }\left(k_{\text {д }}=1\right) ; \\
& W_{\Phi C}(s)=\frac{k_{\Phi C}}{1+s T_{\Phi C}}-\text { передаточная функция формирователя сигналов поворота; } \\
& W_{y M}(s)=\frac{k_{y M}}{1+s T_{\text {УM }}}-\text { передаточная функция усилителя мощности; } \\
& W_{\text {дв }}(s)=\frac{k_{\text {Дв }}}{s\left(1+s T_{\text {Дв }}\right)}-\text { передаточная функция двигателя; } \\
& W_{C u}(s)=\frac{k_{C u}}{1+s T_{C u}}-\text { передаточная функция счетчика угла поворотов; } \\
& W_{K y}(s)=\frac{k_{K V}}{1+s T_{K Y}}-\text { передаточная функция корректирующего устройства в цепи обратной }
\end{aligned}
$$

связи,

где $k_{\Phi П}, k_{д}, k_{\Phi C}, k_{У M}, k_{Д в}, k_{C u}, k_{K V}$ - коэффициенты передачи МФП, дискриминатора (схемы обработки), формирователя сигналов поворота, усилителя мощности, двигателя, счетчика угла поворота, корректирующего устройства в цепи обратной связи соответственно; $T_{\Phi П}, T_{Д}, T_{\Phi C}, T_{y M}$, $T_{Д в}, T_{C \psi}, T_{K У}$ - постоянные времени МФП, дискриминатора (схемы обработки), формирователя сигналов поворота, усилителя мощности, двигателя, счетчика угла поворота, корректирующего устройства в цепи обратной связи соответственно; $i$ - передаточное число редуктора; $s$ - оператор преобразования Лапласа.

Передаточную функцию цепи сигнала ошибки системы сопровождения ЗУР можно записать в виде

$$
W_{c}(s)=\frac{W_{\Phi \Pi}(s) W_{Д}(s) W_{\Phi C}(s) W_{0}(s) / i}{1+W_{\Phi П}(s) W_{\text {Д }}(s) W_{\Phi C}(s) W_{0}(s) / i}
$$

где $W_{0}(s)=\frac{W_{\text {УM }}(s) W_{\not в}(s)}{1+W_{\text {УM }}(s) W_{\text {дв }}(s) W_{K У}(s) W_{C \psi}(s)}-$ передаточная функция внутреннего контура системы, образованной усилителем мощности с двигателем и цепью обратной связи (счетчиком угла поворота, корректирующим устройством). 
Выходной сигнал системы по каналам будет определяться так:

$$
U_{x n}(t)=W_{c}(s) U_{x}(t) ; U_{y n}(t)=W_{c}(s) U_{y}(t) .
$$

Качество работы системы сопровождения ЗУР при случайных воздействиях будем оценивать по динамической ошибке $\Delta U$, представляющей собой разность между текущим напряжением рассогласования $U_{x}\left(U_{y}\right)$ и отработанным системой $U_{x n}\left(U_{y n}\right)$, (обуславливающим текущее положение объектива) на момент времени $t-\Delta U_{x}=U_{x}-U_{x n} ; \Delta U_{y}=U_{y}-U_{y n}$ (см. рис. 1). С учетом выражения (15) динамические ошибки по каналам могут быть определены следующим образом:

$$
\Delta U_{x}(t)=U_{x}(t)\left[1-W_{c}(s)\right] ; \Delta U_{y}(t)=U_{y}(t)\left[1-W_{c}(s)\right]
$$

где $s=\frac{d}{d t}-$ символ дифференцирования.

Разработанная модель была реализована в Matlab Simulink. Результаты моделирования для различных траекторий полета ЗУР и различных помеховых условий в виде графиков представлены на рис. 7.

Система сопровождения работает в условиях действия ряда возмущающих воздействий (помех), основными из которых являются флуктуация отраженного от цели лазерного зондирующего сигнала, внутренние шумы МФП, фоновый шум, а также преднамеренные помехи.

На основании полученных результатов можно сделать следующий вывод: разработанная имитационная модель системы сопровождения атакующей ракеты позволяет обеспечить оценку точности сопровождения. Из анализа результатов моделирования видно, что предлагаемая система сопровождения обеспечивает высокую точность сопровождения атакующей ракеты, но при этом на точность сопровождения существенное влияние оказывает кривизна траектории полета атакующей ракеты и флуктуации изображения ракеты в плоскости фоточувствительной матрицы.

\section{Заключение}

Таким образом, на основе использования лазерно-тепловизионной системы с приемным устройством матричного типа предложен алгоритм высокоточного сопровождения ЗУР по принимаемому изображению. Представлены алгоритмы оценки текущих координат энергетического центра изображения и суммарно-разностной обработки сигналов, осуществляющие измерения углов рассогласования между направлением на ЗУР и оптической осью МФП. Предложен способ повышения помехоустойчивости и чувствительности системы сопровождения на основе применения переменных коэффициентов усиления для элементов матричного фотоприемника. Разработана динамическая модель процесса высокоточного сопровождения, позволяющая оценить эффективность системы при формировании на входе МФП изображения. Проведено исследование алгоритма высокоточного сопровождения ЗУР матричным фотоприемником лазерно-тепловизионной системы в условиях помеховых воздействий. 

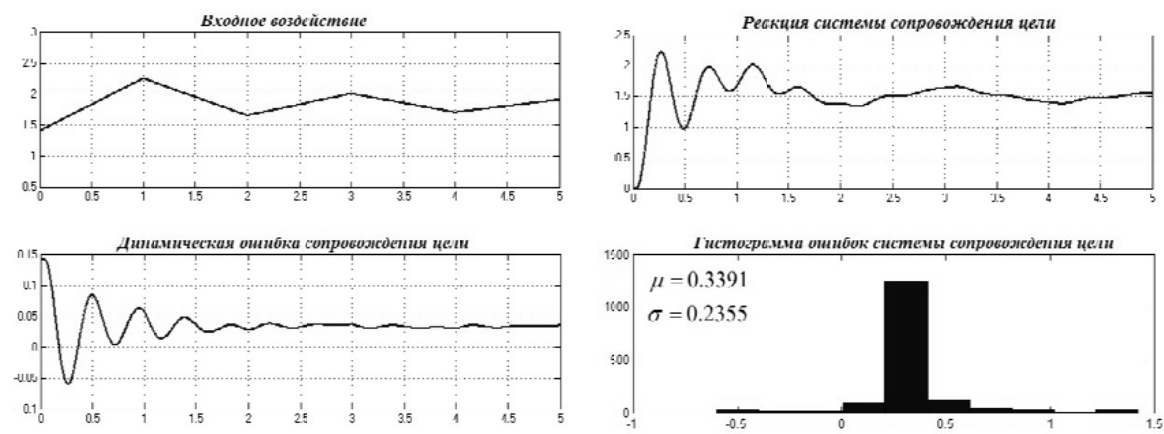

a)
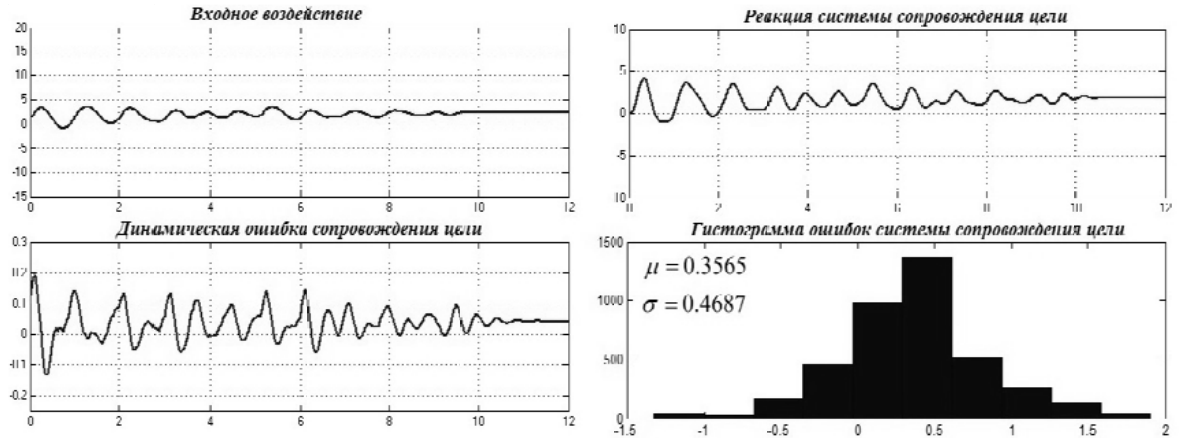

б)
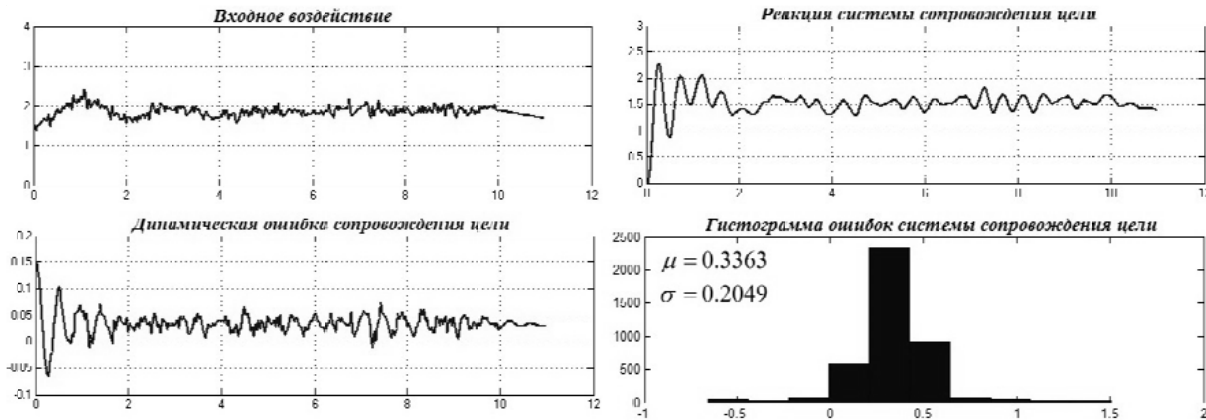

B)
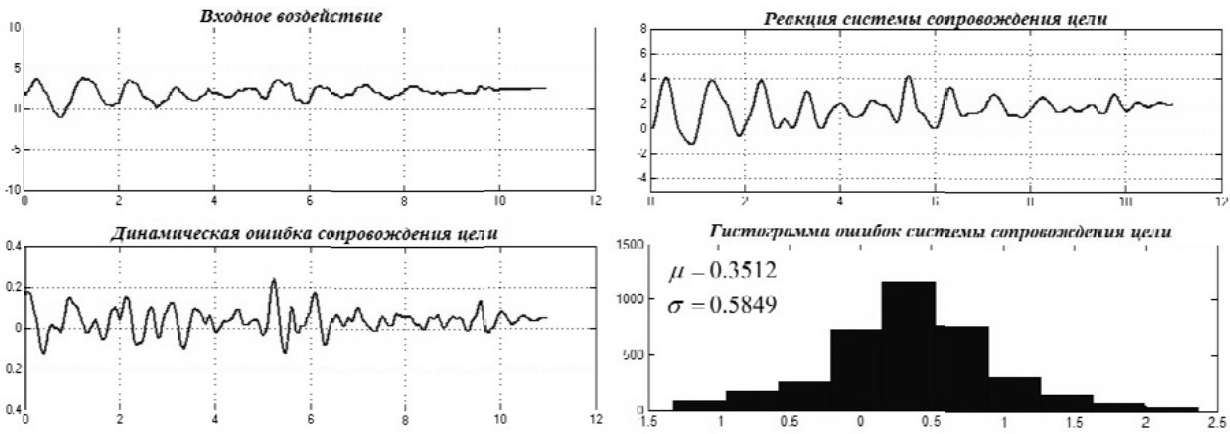

г)

Рис. 7. Результаты исследования эффективности высокоточного сопровождения: $a$ - слабоискривленная траектория; $\sigma$ - сильноискривленная траектория; 6 - слабоискривленная траектория в присутствии шумов; г-сильноискривленная траектория в присутствии шумов 


\section{Список литературы}

[1] Радиоэлектронная борьба. Силовое поражение радиоэлектронных систем / В.Д. Добыкин, А.И. Куприянов, В.Г. Пономарев, Л.Н. Шустов; под ред. А.И. Куприянова. М.: Вузовская книга, 2007. 468 c. [Radio-electronic fight. Power defeat of radio-electronic systems / EL Dobykin, A.I. Kupriyanov, V. G. Ponomarev, L.N. Shustov; Under the editorship of A.I. Kupriyanov. M.: High school book, 2007. 468 p.]

[2] Гальярди Р.М., Карп Ш. Оптическая связь / под ред. А.Г. Шереметьева; пер. с англ. M.: Связь, 1978.[Galyardi R. M., Karp Sh. Optical Communications / Under the editorship of A.G. Sheremetyev; the lane with English - M.: Communication, 1978.]

[3] Криксунов Л.З. Следящие системы с оптико-электронными координаторами. К.: Техника, 1991. 335 с. [Kriksunov L.Z. The watching systems with optical-electronic coordinators. K.: Equipment, 1991. 335 p.]

[4] Заикин В.В. Самонаведение. М.: САЙНС-ПРЕСС.2002. [Zaikin V. V. Self-homing. М.: SAYNS-PRESS.2002.]

[5] Красильников Н.Н. Теория передачи и восприятия изображений. Теория передачи изображений и ее приложения. М.: Радио и связь, 1986. 248 с. [Krasilnikov N. N. Theory of transfer and perception of images. Theory of transfer of images and her appendix. M.: Radio and communication, 1986. 248 р.]

[6] Козирацкий, Ю.Л. Математическая модель координатора управляемого средства доставки с лазерной пассивной системой наведения на излучение опорного источника / Ю.Л. Козирацкий, Д.А. Сербов, М.Л. Паринов. Радиотехника, 2009, 5, 115-117. [Koziratsky, Yu.L. Mathematical model of the coordinator of the operated delivery system with laser passive system of aiming at the radiation of a basic source / Yu.L. Koziratsky, D. A. Serbov, M. L. Parinov//Radio engineering, 2009, 5, 115-117.]

[7] Козирацкий Ю.Л., Прохоров Д.В., Казаков В.С., Кусакин А.В. Алгоритм совместной оценки положения и времени прихода пуассоновского изображения цели и его эффективность 6 условиях помех. Радиотехника. 2012, 5, 102-106. [Koziratsky Yu.L., Prokhorov D.V., Kazakov V. S., Kusakin A.V. Algorithm of a joint assessment of situation and time of arrival of the Poisson image of the purpose and its efficiency in the conditions of hindrances // Radio engineering. M, 2012, 5, 102106]

[8] Обнаружение и координатометрия оптико-электронных средств, оценка параметров их сигналов: монография / под ред. Ю.Л. Козирацкого. М.: Радиотехника, 2015. 456 с. [Detection and koordinatometriya of optical-electronic means, assessment of parameters of their signals. The monograph / Under the editorship of Yu.L. Koziratsky. M.: Radio engineering, 2015. 456 p..]

[9] Козирацкий Ю.Л., Прохоров Д.В., Кусакин А.В. Способ повышения помехоустойчивости алгоритма оценки угловых координат цели матричным приемником. Радиотехника. 2013, 7, 75-77. [Koziratsky Yu.L., Prokhorov D.V., Kusakin A.V. Way of increase of a noise stability of algorithm of an assessment of angular coordinates of the purpose matrix receiver//Radio engineering. M, 2013, 7, 75-77.]

[10]Гонсалес Р., Вудс Р. Цифровая обработка изображений. М.: Техносфера, 2005. 1072 с. [Gonsalez R., Woods River. Digital processing of images. M.: Technosphere, 2005. 1072 p.] 
[11] Коновалов Г.Ф. Радиоавтоматика. М.: Высш. шк., 1990. 335 с. [Konovalov G. F. Radio automatic equipment, M.: graduate School, 1990. 335 p.] 